\title{
Inserting Extra Train Services on High-Speed Railway
}

\author{
Yuyan Tan', Wen Xu', Zhibin Jiang ${ }^{2 *}$, Ziyulong Wang ${ }^{1}$, Bo Sun ${ }^{3}$ \\ ${ }^{1}$ Traffic and Transportation Planning and Management, Department of Transportation Management Engineering, School of \\ Traffic and Transportation, Beijing Jiaotong University, No.3 Shangyuancun, Haidian District, 100044 Beijing, P. R. China \\ 2 College of Transportation Engineering, Tongji University, No.4800 Caoan Road, 201804 Shanghai, P. R. China \\ ${ }^{3}$ Major of Railway Transportation, China Railway Guangzhou Group Corporation, Nr. 151 Zhongshan Road, 510088 Guangzhou, \\ P. R. China \\ * Corresponding author, e-mail: jzb@tongji.edu.cn
}

Received: 27 July 2018, Accepted: 02 September 2019, Published online: 24 January 2020

\begin{abstract}
With the aim of supporting future traffic needs, an account of how to reconstruct an existing cyclic timetable by inserting additional train services will be given in this paper. The Timetable-based Extra Train Services Inserting (TETSI) problem is regarded as an integration of railway scheduling and rescheduling problem. The TETSI problem therefore is considered involving many constraints, such as flexible running times, dwell times, headway and time windows. Characterized based on an event-activity graph, a general Mixed Integer Program model for this problem is formulated. In addition, several extensions to the general model are further proposed. The realworld constraints that concerning the acceleration and deceleration times, priority for overtaking, allowed adjustments, periodic structure and frequency of services are incorporated into the general model. From numerical investigations using data from ShanghaiHangzhou High-Speed Railway in China, the proposed framework and associated techniques are tested and shown to be effective. Keywords

cyclic timetable, extra train services, periodic structure, High-Speed Railway, tolerance of disruption
\end{abstract}

\section{Introduction}

With the development of High-Speed Railway (HSR) in the world, several obvious advantages both in transport marketing and train operation planning are shown by cyclic timetable. In a cyclic timetable, train services are operated regularly with respect to a cycle time (typically one hour), and all the time instants (expressing train departures and arrivals) are expressed modulo this cycle time. Since cyclic timetable are transparent to the customer, there is no need for passengers to memorize complex timetable for their regular connections. Moreover, from the scope of planning, cyclic timetable has the advantage that one only needs to consider one cycle period. The railway operator can focus the planning on one cycle period, not only for the timetable per se. However, for some trains such as the trains with low-frequency and long-distance, they are not suitable to be scheduled as cyclic train paths. Otherwise, it will result in waste of capacity.

The Timetable-based Extra Train Services Inserting (TETSI) problem is considered as an integration of railway scheduling and rescheduling problem. Railway operator both modify the initial trains and make schedules for extra train services. Recently, Train Timetable Scheduling (TTS) and Train Timetable Rescheduling (TTR) problems have been in the limelight. For instance, a list of foremost papers by Törnquist (2006) published on the area of rail timetable optimization between 1980 and 2006, and a recent survey by Hansen (2009) also summarized emerging methods and solution techniques for train timetabling and dispatching. There are varied models are used to formulate timetable scheduling and rescheduling problem.

Extensive researches have been done in the TTS problem in the literatures. By studying on the problem, it consists of the cyclic and non-cyclic versions. Distinctions of model and algorithm are made between scheduling non-cyclic and cyclic timetables. The Periodic Event Scheduling Problem (PESP) is a main basis of most authors that study cyclic timetabling problem, which is introduced by Serafini and Ukovich (1989) and Peeters (2003) considers a PESP based model for the cyclic railway timetabling problem. Regarding the objectives applied, no real dominating objective function could be found but there is a tendency towards minimizing the total travel time and tardiness in Lindner (2000) and 
Schachtebeck (2010). Maximizing robustness, profit, and line's frequency are some other examples. In case of disruptions or disturbance occur, the timetable must be rescheduled to resolve the conflicts. A first Mixed Integer Program (MIP) formulation based on Event-Activity Graph Model is given and further developed in Zhou and Zhong (2005), Nie et al. (2010) and Meng and Zhou (2014). The limited capacity of the track system has been taken into account by Schöbel (2007, 2009). Schmidt (2013) extend this model by considering rerouting of trains. However, adding paths problem are rarely directly discussed about. The only papers to our knowledge are presented as follows. Solving the problem of inserting freights trains with assumption that all of the initial trains cannot be changed by Cacchiani et al. (2014) and Ingolotti et al. (2004). A set of Pareto optimal train schedules with respect to risk and travel time is computed by Flier et al. (2009).

Our study differs from the previous ones in two aspects. Firstly, the realistic constraints of general safety, time window, acceleration and deceleration time, allowable adjustment, periodic structure, flexible speed, station capacity, priority for overtaking, and frequency of services are accounted. Secondly, several objectives are considered, minimizing the total adjustments, maximizing travel speed and robustness.

The paper is compiled as follows:

- In Section 2, the mathematical model for TETSI problem is introduced.

- In Section 3, we apply the proposed model on a High-Speed Railway line in China.

- Section 4 summarizes our results.

\section{Mathematical model}

This section formulates a general Mixed Integer Program (MIP) model for the adding train paths problem. This model is described based on the event-activity graph.

\subsection{Railway network input}

A event-activity graph $G=(V, E)$ is a directed graph whose nodes $V$ are called events and whose directed edges $E$ are called activities, see Fig. 1.

The set of events consists of all arrival events and departure events, i.e. $V=V_{a r r} \cup V_{d e p}$,

$V_{\text {arr }}=\{(t, s$, arrival $):$ train $t$ arrives at station $s\}$

$V_{d e p}=\{(t, s$, departure $):$ train $t$ departs from station $s\}$

The events of set $V$ are linked by directed edge set $E$, which are called activities and consists:

- Trip activities: $E_{\text {trip }} \subset V_{\text {dep }} \times V_{\text {arr }}$ model driving of a train between two consecutive stations.

- Dwell activates: $E_{d w e l l} \subset V_{\text {arr }} \times V_{\text {dep }}$ model the stopping of a train at a station.

- Changing activities: $E_{\text {change }} \subset V_{\text {arr }} \times V_{\text {dep }}$ model a transfer connection from one station to another.

- Headway activities: $E_{\text {headway }} \subset V_{\text {dep }} \times V_{\text {dep }} \times V_{\text {arr }} \times V_{\text {arr }}$ model the security headway between two consecutive departures and arrivals at the same station.

\subsection{Notations}

Table 1 first lists general subscripts and input parameters used in the proposed model. Table 2 describes the decision variables in the proposed optimization model. One minute

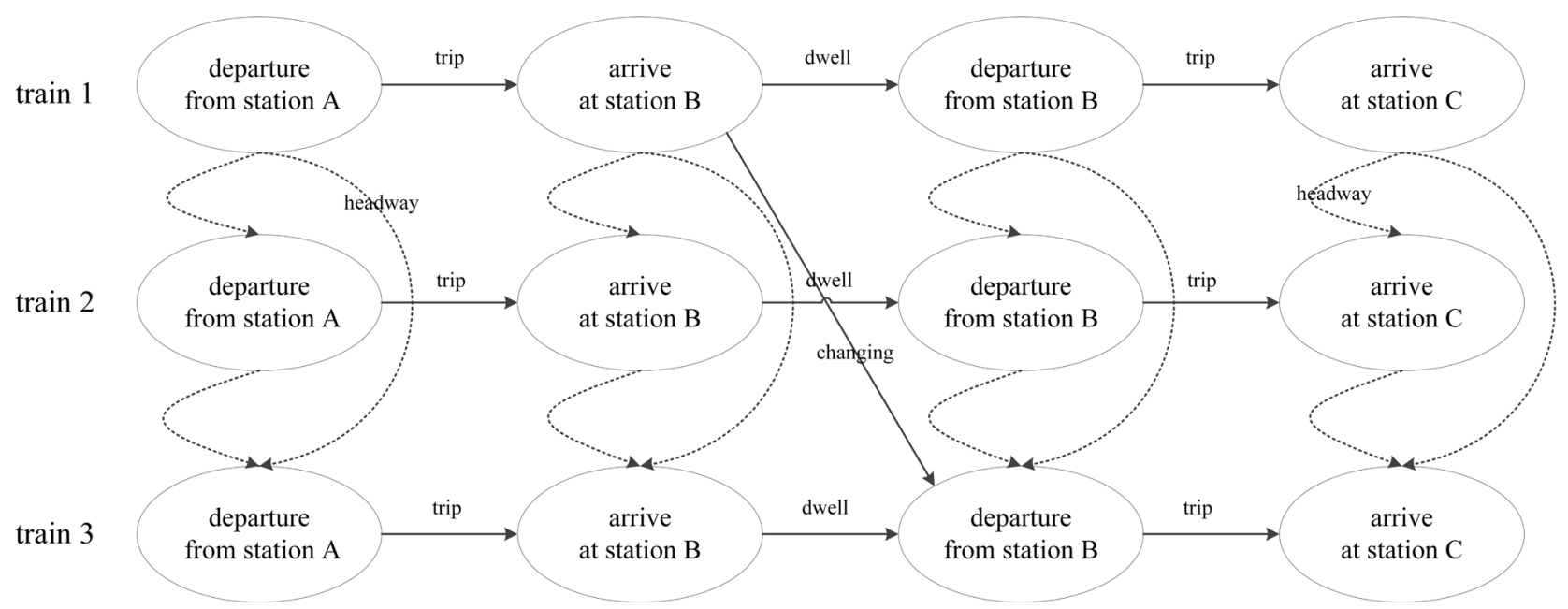

Fig. 1 The event-activity network for railway timetabling 
Table 1 Subscripts and parameters

\begin{tabular}{|c|c|}
\hline Symbol & Description \\
\hline Vadd & set of additional events \\
\hline$V^{i n i}$ & set of initial events \\
\hline$V_{d e p}$ & set of departure events \\
\hline$V_{\text {arr }}$ & set of arrival events \\
\hline$T^{\text {add }}$ & all of the additional trains \\
\hline$T^{n i} i$ & all of the initial trains \\
\hline$E^{\text {add }}$ & set of additional activities \\
\hline$E^{i n i}$ & set of initial activities \\
\hline$i, j$ & event index \\
\hline$e$ & activity index, $e=(i, j)$ \\
\hline$s(i)$ & the station at which event $i$ takes place \\
\hline$b(i, j)$ & the section on which activity $e=(i, j)$ takes place \\
\hline$t(i)$ & the train of event $i$ \\
\hline$\epsilon^{a}$ & required acceleration time \\
\hline$\epsilon^{d}$ & required deceleration time \\
\hline$h$ & the minimum headway time between two consecutive events \\
\hline$p_{i j}$ & $\begin{array}{c}=1 \text { if } \operatorname{train} t(i) \text { has higher priority than } \operatorname{train} t(j), \\
=0 \text { otherwise }\end{array}$ \\
\hline$M$ & a sufficiently large positive integer \\
\hline$\pi_{i}$ & $\begin{array}{c}\text { the time instant at which event } i \in V \text { takes place in the } \\
\text { initial timetable }\end{array}$ \\
\hline$p l s_{i}$ & $\begin{array}{l}=1 \text { if train } t(i) \text { stops at the station } s(i) \text { in the initial timetable, } \\
=0 \text { if train } t(i) \text { bypass the station } s(i) \text { in the initial timetable }\end{array}$ \\
\hline & $\begin{array}{l}\text { the lower bound of the time window at which event } i \\
\text { takes place }\end{array}$ \\
\hline$t w_{i}^{\max }$ & $\begin{array}{l}\text { the upper bound of the time window at which event } i \\
\text { takes place }\end{array}$ \\
\hline trip $_{e}^{\min }$ & the minimum trip time of event $e$ \\
\hline $\operatorname{trip}_{e}^{\max }$ & the maximum trip time of event $e$ \\
\hline$d w e l l_{e}^{\min }$ & the minimum dwell time of event $e$ \\
\hline$d w e l l_{e}^{\max }$ & the maximum dwell time of event $e$ \\
\hline$\Delta$ & maximum allowable adjustment of event \\
\hline$T$ & cyclic time, 1 hour in the paper \\
\hline$N$ & number of additional trains \\
\hline$\theta$ & maximum allowable deviation to periodic structure \\
\hline$\beta$ & bandwidth of frequency \\
\hline$T_{\text {hor }}$ & considered time horizon \\
\hline
\end{tabular}

Table 2 Decision variables

\begin{tabular}{|c|c|}
\hline Symbol & Description \\
\hline$x_{i}$ & $\begin{array}{l}\text { the time instant at which event } i \in V \text { takes place in the } \\
\text { new timetable }\end{array}$ \\
\hline$\lambda_{i j}$ & $\begin{array}{c}=1 \text { if event } j \text { takes place after, or at the same time as event } i, \\
=0 \text { if event } j \text { takes place before event } i\end{array}$ \\
\hline$\rho_{i}$ & $\begin{array}{l}=1 \text { if train } t(i) \text { stops at the station } s(i), \\
=0 \text { if train } t(i) \text { bypass the station } s(i) \text { in the new timetable }\end{array}$ \\
\hline
\end{tabular}

is the unit of all time-related parameters and variables. In this study, we focus on a train timetabling problem on a double-track rail line which consists of a series of uni-directional track segments.

\subsection{Constraints}

The TETSP calls for determining the best schedule for a given set of additional trains based on a given timetable. This schedule of all trains must respect several constraints, which are presented as the following:

- minimum headway times $h$ between consecutive arrivals or departures of trains from a station must be respected for safety;

- minimum dwell times $d$ well of trains at stations must be respected;

- minimum connecting times con for interchanging between two trains must be guaranteed, otherwise the connection would be invalid;

- overtaking of trains in a section is forbidden; and overtaking rule must be satisfied for ensuring a train with higher priority would be overtaken by a lower one;

- frequency constraint is applied to spread the multiple trains of a single train line evenly across the considered time horizon.

As is shown in Fig. 2, the constraints used in the double-track adding paths model are presented as the following:

- Reasonable time window:

$$
\begin{array}{ll}
x_{i} \geq t w_{i}^{\min } & \forall i \in V^{\text {add }} \\
x_{i} \leq t w_{i}^{\max } \quad \forall i \in V^{\text {add }}
\end{array}
$$

- Variable trip time on segment:

$$
\begin{array}{ll}
x_{j}-x_{i} \geq \operatorname{trip}_{e}^{\min }+\rho_{i} * \epsilon^{a}+\rho_{j} * \epsilon^{d} & \forall_{e}=(i, j) \in E_{\text {trip }} \\
x_{j}-x_{i} \leq \operatorname{trip}_{e}^{\max }+\rho_{i} * \epsilon^{a}+\rho_{j} * \epsilon^{d} & \forall_{e}=(i, j) \in E_{\text {trip }}
\end{array}
$$

- Dwell time at station:

$$
\begin{aligned}
& x_{j}-x_{i} \geq \rho_{i} \cdot d w e l l_{e}^{\min } \quad \forall_{e}=(i, j) \in E_{d w e l l} \\
& x_{j}-x_{i} \leq \rho_{i} \cdot d w e l l_{e}^{\max } \quad \forall_{e}=(i, j) \in E_{d w e l l} \\
& \rho_{i}=1 \quad \forall_{i} \in V_{\text {arr }}: p l s_{i}=1
\end{aligned}
$$

- Minimum headway:

$$
x_{j}-x_{i} \geq h_{e} \cdot \lambda_{i j}-M \cdot\left(1-\lambda_{i j}\right) \quad \forall_{e}=(i, j) \in E_{\text {headway }}
$$



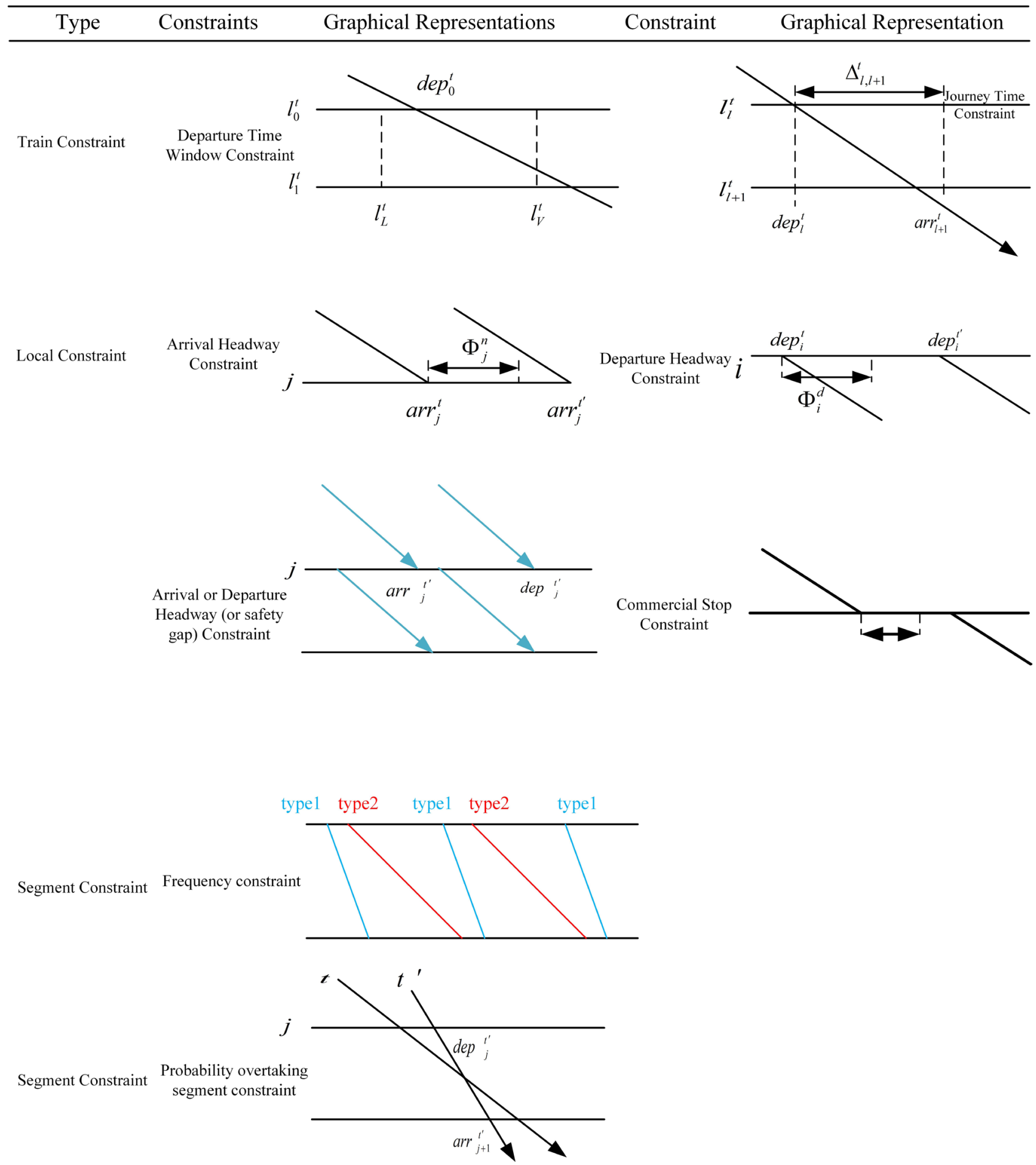

Fig. 2 The constraint of the model

$$
\begin{aligned}
& x_{i}-x_{j} \geq h_{e} \cdot\left(1-\lambda_{i j}\right)-M \cdot \lambda_{i j} \quad \forall_{e}=(i, j) \in E_{\text {headway }} \\
& \lambda_{i j}=\lambda_{i j^{\prime}} \quad \forall b\left(i, i^{\prime}\right)=b\left(j, j^{\prime}\right)
\end{aligned}
$$

- Priority for overtaking:

$$
\lambda_{i j}-\lambda_{i^{\prime} j^{\prime}} \leq 0 \quad \forall\left(i, i^{\prime}\right),\left(j, j^{\prime}\right) \in E_{d w e l l}: s(i)=s(j), p_{i j}=1
$$

$$
\lambda_{i j}-\lambda_{i^{\prime j^{\prime}}}>0 \quad \forall\left(i, i^{\prime}\right),\left(j, j^{\prime}\right) \in E_{d w e l l}: s(i)=s(j), p_{i j}=0
$$

- Adjustment of initial schedules:

$$
\begin{array}{ll}
x_{i}-\pi_{i} \geq a d_{i} & \forall i \in V^{i n i} \\
\pi_{i}-x_{i} \geq a d_{i} & \forall i \in V^{i n i}
\end{array}
$$


- Frequency for additional trains:

$$
\begin{aligned}
& x_{j} \geq x_{i} \quad \forall(i, j) \in E_{s y n}, i, j=1, \ldots, N \\
& x_{j}-x_{i} \geq \frac{j-i}{N-1} T_{h o r}-\beta \quad \forall(i, j) \in E_{s y n}, i, j=1, \ldots, N \\
& x_{j}-x_{i} \leq \frac{j-i}{N-1} T_{h o r}+\beta \quad \forall(i, j) \in E_{s y n}, i, j=1, \ldots, N
\end{aligned}
$$

- Operator preferences:

$$
\begin{aligned}
& x_{i}, a d_{i} \geq 0 \quad \forall i \in V \\
& \rho_{i}, \lambda_{i j} \in\{0,1\} \quad \forall i \in V .
\end{aligned}
$$

Equations (1), (2) represent the reasonable departure time window for trains. Time windows of departure (arrival) times are usually chosen on the board stations. Equations (3), (4) relate the actual trip time on section. Owing to the requirements of safety and passenger comfort, high-speed trains usually take at least several minutes to fully stop or reach a cruise speed even with highly efficient acceleration and deceleration performance in Yang et al. (2010). In this situation, when train stops the corresponding actual trip time has to exactly take into account the required acceleration time $\epsilon^{a}$ and deceleration time $\epsilon^{d}$. As shown in Eqs. (5)-(7), train must stop at all stations at which it calls (i.e. $p l s_{i}=1$, else $p l s_{i}=0$ ). More precisely, extension of a scheduled stop or additional stops is permitted for operational requirements. Equations (8), (9) describe the minimum headway requirements between the departure times and arrival times of consecutive trains at the same station, and Eq. (10) guarantee that trains do not overtake each other on a section. Equations (11), (12) enforce that train of higher priority cannot be overtaken by a lower one. Equations (13), (14) record the magnitude of the right or left shifts $a d_{i}$ of every initial event $i$. Equations (15)-(17) are applied to spread the multiple trains of a single train line evenly across the considered time horizon $T_{h o r}$, which is also known as frequency constraint. In order to formulate this constraint, we introduce a set of frequency activities for every pair of additional trains within a train line.

$$
E_{s y n}=\left\{\begin{array}{l}
(i, j): i \in V^{a d d} \text { and } j \in V^{\text {add }} \\
\text { are scheduled synchronously }
\end{array}\right\}
$$

The parameters $N$ and $\beta$ specify the number of extra trains within a train line and flexibility of the frequency constraint, respectively.

\subsection{Objective functions}

In this paper, we consider objectives in the view of the following three aspects,

1. high quality of the performance to the additional trains, which can be represented by the objective, to minimize travel time of additional trains $F_{t}=\sum_{t \in T^{\text {add }}}\left(x_{\text {last }_{t}}-x_{\text {first }_{t}}\right) / N$

2. low deviations to the initial services, which can be represented by the objective, to minimize the total adjustments to initial trains $F_{a}=\sum_{i \in v^{i t}} a d_{i}$

3. here, first $t_{t}$ and last $_{t}$ are the first and last event of train $t$ respectively. $a d_{i}$ is an auxiliary variable,

$$
\begin{aligned}
& x_{i}-\pi_{i} \leq a d_{i} \quad i \in V^{i n i} \\
& \pi_{i}-x_{i} \leq a d_{i} \quad i \in V^{i n i}
\end{aligned}
$$

4. maximizing the robustness of the new timetable $F_{r}$.

We will describe the function of robustness in the TETSI problem in more detail. The timetable robustness can be improved by pulling apart trains that share a track. If there is a lot of times between two consecutive trains, these times can be used as buffers in case of delays. Peeters (2003) modelled a robustness cyclic timetable by setting the interval of trains be closed to the middle of the time window in order to pull apart each other. For the robustness in the TETSI problem, a trade-off has to be made, however, between increasing the interval time between trains on one hand and decreasing the modifications to initial timetable on the other hand. For example, during inserting additional trains, although the involved trains share the track for entering or leaving the station, the requirement of minimizing deviations to initial timetable implies that these trains cannot be pulled apart too far.

Then the objective of robustness in the TETSI problem restraints that an additional train should be inserted in the position that

- between two trains that of largest idle interval, and simultaneously,

- on or be closed to the middle of the interval time.

One particular kind of this situation as an example is shown in Fig. 3. Three existing trains $t_{1}, t_{2}$ and $t_{3}$ are scheduled in the initial timetable, and a new train needs to be inserted. Both arrival and departure headway are set to be $3 \mathrm{~min}$. The additional train could be inserted between $t_{2}$ 


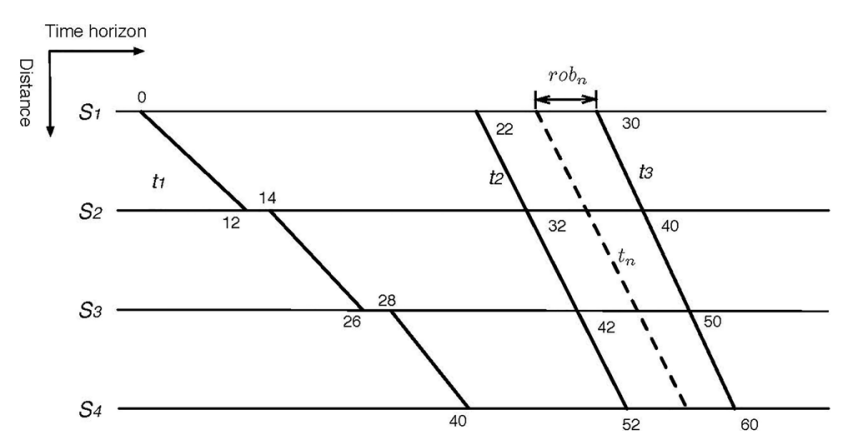

(a)

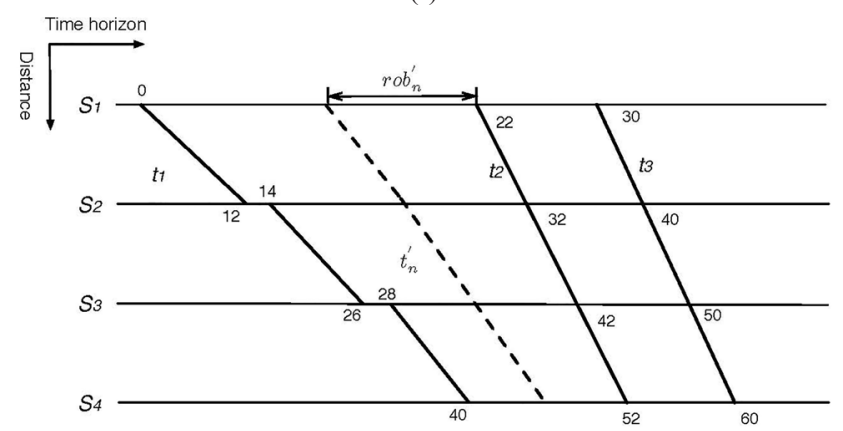

(b)

Fig. 3 Different robustness results from different insertion

(a) The additional train is inserted between $t_{1}$ and $t_{2}$

(b) The additional train is inserted between $t_{2}$ and $t_{3}$

and $t_{3}$ as train path $t_{n}$ shown in Fig. 3 (a), or $t_{1}$ and $t_{2}$ as train path $t_{n}^{\prime}$ shown in Fig. 3 (b). Both of the solutions do not lead any deviation to initial services. However, the robustness of the new timetables are completely different, in practice timetable in Fig. 3 (b) is preferred due to a better robustness. Merely minimizing the modifications or trip times does not suit our goal to get a robustness insertion.

Let $E_{\text {headway }}^{r}=(v, u)$ be the set of headway activities corresponding to the safety constraints, where $v \in V^{a d d}, u \in V$. Then, the departure times of trains are pulled apart when the process time for $e \in E_{\text {headway }}^{r}$ is increased, i.e. the additional trains are inserted in the middle of the adjacent trains between which there has the largest time interval.

Therefore, introduce an auxiliary variable $\gamma_{e}$ for all $e \in E_{\text {headway }}^{r}$. The auxiliary variable $\gamma_{e}$ is constrained as

$\gamma_{e} \geq x_{i}-x_{j} \quad \forall_{e}=(i, j) \in E_{\text {headway }}^{r}$

$\gamma_{e} \geq x_{j}-x_{i} \quad \forall_{e}=(i, j) \in E_{\text {headway }}^{r}$

so $\gamma_{e} \geq\left|x_{i}-x_{j}\right|$. Then define the parameter $\operatorname{rob}_{i}$ as

$\operatorname{rob}_{i}=\min \gamma_{e} \quad \forall_{e}=(i, j) \in E_{\text {headway }}^{r}$.

That is, $r o b_{i}$ denotes the minimum time interval between the additional activity $i$ and other activities. Thus, maximizing $r o b_{i}$ means pushing $x_{i}$ away from the other trains, and thus insert the additional trains in the middle of largest time interval.

Using the above, the robustness objective function in defined as

$F_{r}=\sum_{i \in V^{\text {add }}} r o b_{i}$.

Recall that the function $F_{r}$ is maximized. This ensures that the additional trains are inserted in the middle of largest time intervals. In other words, maximizing $F_{r}$ means maximizing the new timetable robustness.

\section{Application on the High-Speed Railway line in China}

The formulation has been applied to Shanghai-Hangzhou HSR in China. This rail line consists of double-tracked HSR lines that 9 major stations. The cyclic nature of the timetable is demonstrated in Fig. 4. The traffic data of an initial daily cyclic timetable includes 159 trains.

The additional trains are inserted while taking the structure of the planned cyclic timetable into account. In a cyclic timetable, train connections are operated regularly with respect to a cycle time. During the process of adding and adjusting the schedules of trains, one usually runs into problems that the periodicity of initial cyclic timetable might be ruined. In order to fully take the advantage of cyclic timetable, the periodic pattern of initial trains is desired to be guaranteed. However, sometimes we do not want to fix the initial timetable too much beforehand. Therefore, the model requires the departure times to be a period time (usually 1 hour) apart, with a bandwidth of $\theta$ minutes.

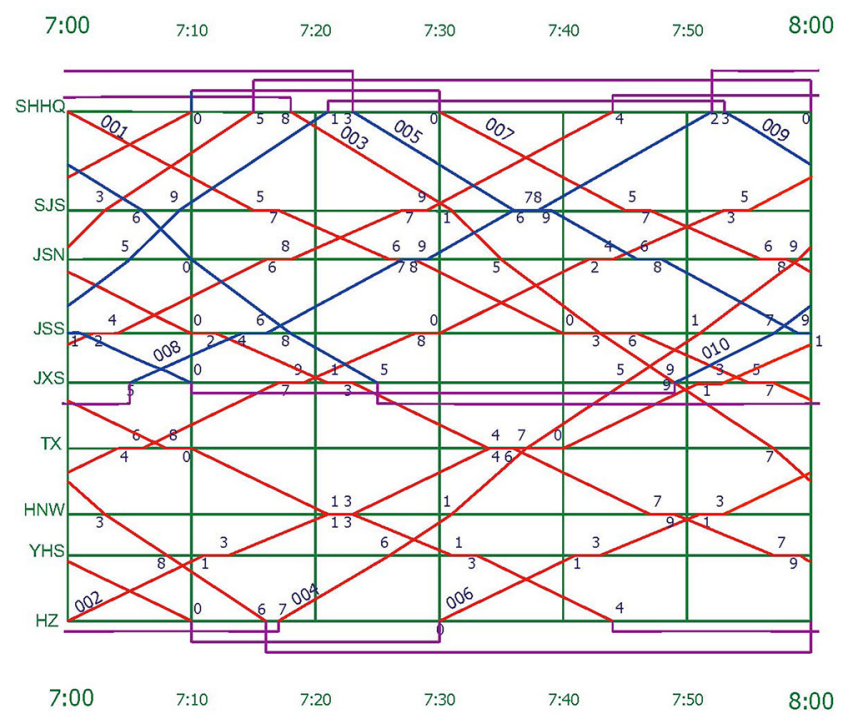

Fig. 4 One-hour time-space diagram for the track between Shanghai and Hangzhou 
For the experiments, we inserted 10 additional trains with a fixed frequency $\beta=10$. The existing timetable may be adjusted under the different tolerance of disruptions. However, what an acceptable level of disruption is however is fairly subjective. For the adding paths problem described in this paper, the tolerance of disruption for initial cyclic timetable can be constrained by,

1. Allowed adjustments $\Delta$, which implies that only a certain amount of left or right shift are allowed for initial trains.

2. Allowed deviations to the periodic structure $\theta$.

Table 3 shows a complete description of the instances constructed using various allowed adjustment $\Delta$ and periodic structure $\theta$. Also, these 20 instances are successively assessed with the four objective functions described above.

There is a trade-off between the minimum total adjustments and minimum trip time. To evaluate the impact of different objectives on the total adjustments, two scenarios of instances, each one emphasizing one aspect of the objective function, have been considered. The scenarios are:

- Scenario 1: Emphasize minimization of average trip time $F_{t}$;

- Scenario 2: Balance adjustments and minimizing of trip time $w_{t} F_{t}+w_{a} F_{a}$.

Table 3 Set of instances with different tolerance of disruptions

\begin{tabular}{|c|c|c|}
\hline Instance Nr. & $\Delta(\min )$ & $\theta$ (min) \\
\hline 1 & 1 & 0 \\
\hline 2 & 1 & 1 \\
\hline 3 & 2 & 0 \\
\hline 4 & 2 & 1 \\
\hline 5 & 2 & 2 \\
\hline 6 & 3 & 0 \\
\hline 7 & 3 & 1 \\
\hline 8 & 3 & 2 \\
\hline 9 & 3 & 3 \\
\hline 10 & 4 & 0 \\
\hline 11 & 4 & 1 \\
\hline 12 & 4 & 2 \\
\hline 13 & 4 & 3 \\
\hline 14 & 4 & 4 \\
\hline 15 & 5 & 0 \\
\hline 16 & 5 & 1 \\
\hline 17 & 5 & 2 \\
\hline 18 & 5 & 3 \\
\hline 19 & 5 & 4 \\
\hline 20 & 5 & 5 \\
\hline
\end{tabular}

The coefficients $w_{t}$ and $w_{a}$ are introduced to permit some increase of trip time if adjustments become too large.

- Scenario 3: Emphasize maximization of robustness $F_{r}$;

- Scenario 4: Balance adjustments and robustness $w_{r} F_{r}+w_{a} F_{a}$.

The coefficients $w_{r}$ and $w_{a}$ are introduced to permit some decrease of robustness if adjustments become too large.

In Tables 4 and 5, comparisons of the result for each instance are reported. The third column is the objective value corresponding to various objective functions. The fourth column shows the total adjustments in minutes to the initial timetable. The arrows and numbers in parenthesis is the relative changes between the emphasizing objective function and its corresponding balance objective function. The fifth Column is average trip time or robustness for four scenarios, respectively. The arrows and numbers in parenthesis is the relative changes too.

The result of Table 4 and 5 highlight the impact of the different objective functions on the total adjustments when the initial timetable is unfixed. These tables present the optimal results depending on the type of objectives the emphasis is put on. On one hand, by introducing the balance objectives, the value of trip time and robustness is not significantly affected, but the value of total adjustments is decreased dramatically. This implies that a balance objective which takes the adjustments into account in more appropriate in practice. On the other hand, the higher level of disruption tolerance to initial timetable also lead to more CPU times. This is caused by more chances of insertions and adjustments. In addition, achieving the balance objectives is more time consumed than the emphasizing objectives. However, the consumed times in all of the instances are acceptable for a tactical or short-term planning.

\section{Conclusion}

In this paper, we model the TETSI problem with several additional real-world constraints, such as the frequency constraint, and the tolerance of adjustments, especially the violation of periodic structure to the initial cyclic timetable. They are considered in light of the practical concerns. Firstly, the deviations to initial timetable are limited, otherwise it will turn to a timetabling problem which has been extensive studied in previous literatures. Secondly, the frequency constraint guarantees the regular train services instead of concentrated distributions. The various objective functions have also been demonstrated and analyzed. 
Table 4 Result of Scenario 1 and Scenario 2

\begin{tabular}{|c|c|c|c|c|c|}
\hline & $\begin{array}{c}\text { Instance } \\
\mathrm{Nr} .\end{array}$ & $\begin{array}{c}\text { Objective } \\
\text { value }\end{array}$ & $\begin{array}{c}\text { Total } \\
\text { adjustment }\end{array}$ & $\begin{array}{l}\text { Trip } \\
\text { time }\end{array}$ & $\begin{array}{l}\mathrm{CPU} \\
\text { time } \\
(\mathrm{s})\end{array}$ \\
\hline \multirow{20}{*}{$\begin{array}{l}\text { Scenario 1: } \\
\text { Emphasize } \\
\text { minimization } \\
\text { of average trip } \\
\text { time }\end{array}$} & 1 & 91 & 1058 & 91 & 6 \\
\hline & 2 & 90 & 1049 & 90 & 6 \\
\hline & 3 & 90 & 2082 & 90 & 12 \\
\hline & 4 & 90 & 1902 & 90 & 16 \\
\hline & 5 & 90 & 2018 & 90 & 14 \\
\hline & 6 & 89 & 2657 & 89 & 14 \\
\hline & 7 & 89 & 3036 & 89 & 21 \\
\hline & 8 & 89 & 2777 & 89 & 20 \\
\hline & 9 & 89 & 3003 & 89 & 25 \\
\hline & 10 & 89 & 3674 & 89 & 12 \\
\hline & 11 & 89 & 3919 & 89 & 12 \\
\hline & 12 & 89 & 4173 & 89 & 13 \\
\hline & 13 & 89 & 4418 & 89 & 13 \\
\hline & 14 & 89 & 4371 & 89 & 13 \\
\hline & 15 & 89 & 4747 & 89 & 19 \\
\hline & 16 & 89 & 5078 & 89 & 30 \\
\hline & 17 & 89 & 4574 & 89 & 20 \\
\hline & 18 & 89 & 4697 & 89 & 36 \\
\hline & 19 & 89 & 5549 & 89 & 27 \\
\hline & 20 & 89 & 5242 & 89 & 43 \\
\hline \multirow{20}{*}{$\begin{array}{l}\text { Scenario 2: } \\
\text { Balance } \\
\text { adjustments } \\
\text { and } \\
\text { minimization } \\
\text { of trip time }\end{array}$} & 1 & 2750 & 14 & 91 & 21 \\
\hline & 2 & 2730 & 24 & 90 & 14 \\
\hline & 3 & 2750 & 14 & 91 & 31 \\
\hline & 4 & 2730 & 24 & 90 & 31 \\
\hline & 5 & 2726 & 26 & 90 & 30 \\
\hline & 6 & 2750 & 14 & 91 & 39 \\
\hline & 7 & 2730 & 24 & 90 & 45 \\
\hline & 8 & 2726 & 26 & 90 & 40 \\
\hline & 9 & 2726 & 26 & 90 & 61 \\
\hline & 10 & 2750 & 26 & 91 & 44 \\
\hline & 11 & 2730 & 14 & 90 & 50 \\
\hline & 12 & 2726 & 24 & 90 & 57 \\
\hline & 13 & 2726 & 26 & 90 & 89 \\
\hline & 14 & 2726 & 26 & 90 & 78 \\
\hline & 15 & 2750 & 26 & 91 & 72 \\
\hline & 16 & 2730 & 14 & 90 & 61 \\
\hline & 17 & 2726 & 24 & 90 & 75 \\
\hline & 18 & 2726 & 26 & 90 & 96 \\
\hline & 19 & 2726 & 26 & 90 & 102 \\
\hline & 20 & 2726 & 26 & 90 & 125 \\
\hline
\end{tabular}

In ongoing and future research, our model and solution approach will to a larger extent be evaluated in a practical context and analyzed in cooperation with the traffic managers and operators. Another practical consideration that has to be taken into account in the future is the consideration
Table 5 Result of Scenario 3 and Scenario 4

\begin{tabular}{|c|c|c|c|c|c|}
\hline & $\begin{array}{c}\text { Instance } \\
\mathrm{Nr} .\end{array}$ & $\begin{array}{l}\text { Objective } \\
\text { value }\end{array}$ & $\begin{array}{c}\text { Total } \\
\text { adjustment }\end{array}$ & Robustness & $\begin{array}{c}\mathrm{CPU} \\
\text { time } \\
\text { (s) }\end{array}$ \\
\hline \multirow{20}{*}{$\begin{array}{l}\text { Scenario 3: } \\
\text { Emphasize } \\
\text { maximi- } \\
\text { zation of } \\
\text { robustness }\end{array}$} & 1 & 136 & 996 & 136 & 37 \\
\hline & 2 & 141 & 1026 & 141 & 34 \\
\hline & 3 & 137 & 1733 & 137 & 49 \\
\hline & 4 & 143 & 1862 & 143 & 101 \\
\hline & 5 & 148 & 1801 & 148 & 127 \\
\hline & 6 & 141 & 2552 & 141 & 40 \\
\hline & 7 & 146 & 2392 & 146 & 76 \\
\hline & 8 & 152 & 2462 & 152 & 103 \\
\hline & 9 & 157 & 2412 & 157 & 79 \\
\hline & 10 & 144 & 2928 & 144 & 58 \\
\hline & 11 & 150 & 3125 & 150 & 89 \\
\hline & 12 & 155 & 2726 & 155 & 183 \\
\hline & 13 & 161 & 3015 & 161 & 117 \\
\hline & 14 & 166 & 2877 & 166 & 439 \\
\hline & 15 & 146 & 3183 & 146 & 40 \\
\hline & 16 & 153 & 3272 & 153 & 175 \\
\hline & 17 & 158 & 3740 & 158 & 136 \\
\hline & 18 & 164 & 3207 & 164 & 238 \\
\hline & 19 & 169 & 3119 & 169 & 316 \\
\hline & 20 & 174 & 3988 & 174 & 101 \\
\hline \multirow{20}{*}{$\begin{array}{l}\text { Scenario 4: } \\
\text { Balance } \\
\text { adjust- } \\
\text { ments and } \\
\text { robustness } \\
\text { trip time }\end{array}$} & 1 & 2648 & 72 & 136 & 62 \\
\hline & 2 & 2790 & 30 & 141 & 68 \\
\hline & 3 & 2648 & 72 & 136 & 54 \\
\hline & 4 & 2790 & 30 & 141 & 200 \\
\hline & 5 & 2903 & 57 & 148 & 132 \\
\hline & 6 & 2648 & 72 & 136 & 109 \\
\hline & 7 & 2790 & 30 & 141 & 197 \\
\hline & 8 & 2903 & 57 & 148 & 193 \\
\hline & 9 & 3003 & 137 & 157 & 194 \\
\hline & 10 & 2648 & 72 & 163 & 139 \\
\hline & 11 & 2790 & 30 & 136 & 304 \\
\hline & 12 & 2903 & 57 & 141 & 129 \\
\hline & 13 & 3003 & 137 & 148 & 282 \\
\hline & 14 & 3090 & 170 & 157 & 292 \\
\hline & 15 & 2648 & 72 & 163 & 102 \\
\hline & 16 & 2790 & 30 & 170 & 308 \\
\hline & 17 & 2903 & 57 & 90 & 234 \\
\hline & 18 & 3003 & 137 & 90 & 587 \\
\hline & 19 & 3090 & 170 & 90 & 234 \\
\hline & 20 & 3162 & 238 & 90 & 503 \\
\hline
\end{tabular}

of multiple objectives. We have seen several evaluation criteria of practical relevance for an insertion, including minimization of adjustments, and minimization of travel time. Our model considers the multi-objective combined models with an objective function being the weighted 
sum of the original objective functions. How to define and use suitable parameters in the objective function to represent the trade-off between various criteria still need to be discussed in detail. In addition, all of the initial trains have the same value of penalty to be adjusted in this paper. Applying various penalties to high-speed and middle-speed trains for example may lead to middle-speed trains becoming less prioritized than high-speed trains. Furthermore, other principle approaches for considering multiple objectives simultaneously, for instance, Pareto optimal solutions, may be considered.

\section{References}

Cacchiani, V., Huisman, D., Kidd, M., Kroon, L., Toth, P., Veelenturf, L., Wagenaar, J. (2014) "An overview of recovery models and algorithms for real-time railway rescheduling", Transportation Research Part B: Methodological, 63, pp. 15-37. https://doi.org/10.1016/j.trb.2014.01.009

Flier, H., Graffagnino, T., Nunkesser, M. (2009) "Scheduling Additional Trains on Dense Corridors", In: Vahrenhold, J. (ed.) Experimental Algorithms, Lecture Notes in Computer Science, vol. 5526, Springer, Berlin, Heidelberg, Germany, pp. 149-160. https://doi.org/10.1007/978-3-642-02011-7_15

Hansen, I. A. (2009) "Railway Network Timetabling and Dynamic Traffic Management", In: 2nd International Conference on Recent Advances in Railway Engineering (ICRARE-2009), Iran university of science and Technology, Tehran, I.R. Iran, pp. 135-145.

Ingolotti, L., Barber, F., Tormos, P., Lova, A., Salido, M., Abril, M. (2004) "An Efficient Method to Schedule New Trains on a Heavily Loaded Railway Network", In: Lemaître, C., Reyes, C. A., González, J. A. (eds.) Advances in Artificial Intelligence - IBERAMIA 2004, Lecture Notes in Computer Science, vol. 3315, Springer, Berlin, Heidelberg, Germany, pp. 164-173. https://doi.org/10.1007/978-3-540-30498-2_17

Peeters, L. W. P. (2003) "Cyclic Railway Timetable Optimization", PhD Thesis, Erasmus Universiteit Rotterdam.

Lindner, T. (2000) "Train Schedule Optimization in Public Rail Transport", PhD Dissertation, Technische Universität Braunschweig.

Meng, L., Zhou, X. (2014) "Simultaneous train rerouting and rescheduling on an N-track network: A model reformulation with network-based cumulative flow variables", Transportation Research Part B: Methodological, 67, pp. 208-234.

https://doi.org/10.1016/j.trb.2014.05.005

Nie, L., Fei, D. B., Zhou, S. D., Fu, H. L., Tong, L. (2010) "Key Issues on Train Line Planning for Beijing-Shanghai High Speed Railway", In: 2010 Joint Rail Conference, vol. 2, Urbana, Illinois, USA, pp. 435-440.

https://doi.org/10.1115/JRC2010-36165

\section{Acknowledgement}

The project presented in this article is supported by Fundamental Research Funds for the Central Universities (No. 2018JBM027), the 111 Project of China (No. B18004), the National Key Research and Development Program of China (No. 2018YFB1201402), and the National Natural Science Foundation of China (Grant Nos. U1434207, U1734204).

Serafini, P., Ukovich, W. (1989) "A Mathematical Model for Periodic Scheduling Problems", SIAM Journal on Discrete Mathematics, 2(4), pp. 550-581. https://doi.org/10.1137/0402049

Schachtebeck, M. (2010) "Delay Management in Public Transportation: Capacities, Robustness, and Integration", Ph.D. Dissertation, Georg-August-Universität Göttingen.

Schmidt, M. (2013) "Simultaneous optimization of delay management decisions and passenger routes", Public Transport, 5(1-2), pp. 125-147. https://doi.org/10.1007/s12469-013-0069-5

Schöbel, A. (2007) "Integer Programming Approaches for Solving the Delay Management Problem", In: Geraets, F., Kroon, L., Schoebel, A., Wagner, D., Zaroliagis, C. (eds.) Algorithmic Methods for Railway Optimization, Lecture Notes in Computer Science, vol. 4359, Springer, Berlin, Heidelberg, Germany, pp. 145-170. https://doi.org/10.1007/978-3-540-74247-0_7

Schöbel, A. (2009) "Capacity constraints in delay management", Public Transport, 1(2), pp. 135-154.

https://doi.org/10.1007/s12469-009-0010-0

Törnquist, J. (2006) "Computer-based decision support for railway traffic scheduling and dispatching: A review of models and algorithms", In: Kroon, L. G., Möhring, R. H. (eds.) 5th Workshop on Algorithmic Methods and Models for Optimization of Railways (ATMOS'05), OpenAccess Series in Informatics (OASIcs), vol. 2, Schloss Dagstuhl - Leibniz-Zentrum für Informatik, Saarbrücken/ Wadern, Germany, pp. 1-23. https://doi.org/10.4230/OASIcs.ATMOS.2005.659

Yang, D., Nie, L., Tan, Y., He, Z., Zhang, Y. (2010) "Working out an incomplete cyclic train timetable for high-speed railways by computer", WIT Transactions on The Built Environment, 114, pp. 889-900. https://doi.org/10.2495/CR100801

Zhou, X., Zhong, M. (2005) "Bicriteria train scheduling for high-speed passenger railroad planning applications", European Journal of Operational Research, 167(3), pp. 752-771. https://doi.org/10.1016/j.ejor.2004.07.019 